Historic, Archive Document

Do not assume content reflects current scientific knowledge, policies, or practices. 



\section{NATIVE TREES, SHRUBS AND WOODY VINES GROWING IN GRANBY, CONN., AND VICINITY}

\begin{tabular}{|c|c|c|}
\hline White Oak & Quercus alba & L. \\
\hline Swamp White Oak & Quercus bicolor & Willd. \\
\hline Chestnut Oak & Quercus Prinus & L. \\
\hline Scarlet Oak & Quercus Coccinea & Wang. \\
\hline Black Oak & Quercus tinctoria & Bartram. \\
\hline Red Oak & Quercus rubra & L. \\
\hline Pin Oak & Quercus palustris & Du Roi. \\
\hline Black Scrub Oak & Quercus ilicifolia & Wang. \\
\hline Dwarf Chestnut Oak & Quercus humilis & Marsh. \\
\hline Chestnut & Castana Vesca & L. \\
\hline American Beach & Fagus ferruginea & Ait. \\
\hline Hop Hornbeam & Ostrya Virginica, & Willd. \\
\hline Blue Beach & Carpinus Americana & Michx. \\
\hline Black Birch & Betula lenta & L. \\
\hline Yellow Birch & Betula lutea & Michx. \\
\hline White Birch & Betula alba & Spach. \\
\hline Paper Birch & Betula papyracea & Ait. \\
\hline Large Toothed Aspen & Populus grandidentata & Michx: \\
\hline American Aspen & Populus tremuloides & Michx. \\
\hline Sugar Maple & Acer saccharinum & Wang. \\
\hline Silver Maple & Acer dasycarpum & Ehrhart \\
\hline Red Maple & Acer rubrum & L. \\
\hline Mountain Maple & Acer spicatum & Lam. \\
\hline Striped Maple & Acer Pennsylvanicum & L. \\
\hline Red Ash & Fraxinus Pubescens & Lam. \\
\hline White Ash & Fraxinus Americana & $\mathrm{L}$. \\
\hline Black Ash & Fraxinus sambucif olia & Lam. \\
\hline Sycamore & Platanus occidentalis & L. \\
\hline Butternut & Juglans cinerea & L. \\
\hline Black-Walnut & Juglans nigra & L. \\
\hline Shellbark Hickory & Carya alba & Nutt. \\
\hline Whitehearted Hickory & Carya tomentosa & Nutt. \\
\hline Brown Hickory & Carya porcina & Nutt. \\
\hline Swamp Hickory & Carya amara & Nutt. \\
\hline Pepperidge & Ny'ssa multiflora & Wang. \\
\hline Mountain Ash & Pyrus Americana & D. C. \\
\hline Choke Berry & Pyrus arbutifolia & L. \\
\hline Dog Berry & Pyrus nigra & Marsh. \\
\hline Shadbush & Amelanchier Canadensis & Torry \& Gray. \\
\hline Wild Vellow Plum & Prunus Americana & Marshall \\
\hline Choke Cherry & Prunus Virginiana & L. \\
\hline Wild Red Cherry & Prunus Pennsylvanica & L. \\
\hline Black Cherry & Prunus serotina & Ehrhart \\
\hline Dwarf Cherry & Prunus pumila & $\mathrm{L}$. \\
\hline White Pine & Pinus Strobus & L. \\
\hline Red Pine & Pinus resinosa & Ait. \\
\hline Pitch Pine & Pinus ridiga & Miller \\
\hline Black Spruce & Abies nigra & Poir. \\
\hline Hemlock & Abies Canadensis & Michx. \\
\hline Hackmatack & Larix Americana & Michx. \\
\hline Juniper & Juniperus communis & L. \\
\hline Red Cedar & Juniperus Virginiana & L. \\
\hline Ground Hemlock & Taxus Canadensis & Willd. \\
\hline Hackberry & Celtis occidentalis & L. \\
\hline Red Elm & Ulmus fulva & Mich. \\
\hline White EIm & Ulmus Americana & L. \\
\hline Red Mulberry & Morus rubra & L. \\
\hline Speckled Alder & Alnus incana & Willd. \\
\hline Smooth Alder & Alnus serrulata & Ait. \\
\hline Heartleaved Willow & Salix cordata & Muhl. \\
\hline Glaucus Willow & Salix discolor & Muhl. \\
\hline Silky Willow & Salix sericea & Marshall \\
\hline Prairie Willow & Salix humilis & Marshall \\
\hline Bibb's Willow & Salix rostrata & Richards \\
\hline Dwarf Gray Willow & Salix tristis & Ait. \\
\hline Hoary Willow & Salix candida & Willd. \\
\hline Black Willow & Salix falcata & Pursh. \\
\hline Sassafras & Sassafras officinale & Nees. \\
\hline Witch Hazel & Hamamelis Virginica & L. \\
\hline Tulip Tree & Liriodendron Tulipifera & L. \\
\hline Basswood & Tilia Americana & L. \\
\hline Prickly Ash & Zanthoxylum Americanum & Mill. \\
\hline Staghorn Sumach & Rhus typhina & L. \\
\hline Smooth Sumach & Rhus Glabra & L. \\
\hline
\end{tabular}

\begin{tabular}{|c|c|c|}
\hline Dwarf Sumach & Rhus copallina & L. \\
\hline Poison Ivy & Rhus Toxicodendron & L. \\
\hline Poison Sumach & Rhus Venenata & D. C. \\
\hline Fox Grape & Vitis Labrusca & L. \\
\hline Summer Grape & Vitis æstivalis & Michx. \\
\hline Frost Grape & Vitis cordifolia & Michx. \\
\hline Virginian Creeper & Ampelopsis quinquefolia & Michx. \\
\hline Red Root & Ceanothus Americanus & L. \\
\hline Bladder Nut & Staphylea trifolia & L. \\
\hline Meadow Sweet & Spiræa salicifolia & L. \\
\hline Hardhack & Spiræa tomentosa & $\mathrm{L}$. \\
\hline Scarlet Fruited Thorn & Cratægus coccinea & L. \\
\hline Cockspur Thorn & Cratægus Crus-galli & L. \\
\hline Wild Gooseberry & Ribes Cynosdati & L. \\
\hline Wild Black Currant & Ribes floridum & L. \\
\hline Flowering Dogwood & Cornus Florida & L. \\
\hline Round Leaved Dogwood & Cornus Circinata & L. Fer. \\
\hline Silky Cornus & Cornus sericea & L. \\
\hline Red Osier & Cornus stolonifera & Michx. \\
\hline Panicled Dogwood & Cornus paniculata & L. Hea. \\
\hline Alternate Leaved Dogwood & Cornus alternifolia & L. \\
\hline Dwarf Cornel & Cornus Canadensis & L. \\
\hline Redberried Elder & Sambucus pubens & Michx. \\
\hline Common Elder & Sambucus Canadensis & L. \\
\hline Button Bush & Cephalanthus occidentalis & L. \\
\hline Sweet Viburnum & Viburnum Lentago & L. \\
\hline Arrow Wood & Viburnum dentatum & L. \\
\hline Maple Leaved Arrow Wood & Viburnum acerifolium & L. \\
\hline Hobble Bush & Viburnum lantanoides & Michx. \\
\hline Cranberry Bush & Viburnum Opulus & L. \\
\hline Downy Arrow Wood & Viburnum pubescens & Push. \\
\hline With-rod & Viburnum cassinoides & L. \\
\hline Black Huckleberry & Gaylussacia resinosa & Torr. \& Gray. \\
\hline Dangleberry & Gay-lussacia frondosa & Torr. \& Gray. \\
\hline Dwarf Blueberry & Vaccinum Pennsylvanicum & Lam. \\
\hline Blueberry & Vaccinum vacillans & Sabads. \\
\hline Swamp Blueberry & Vaccinum corymbosum & L. \\
\hline Leather Leaf & Cassandra caly culata & Don. \\
\hline Rosemerry & Andromeda polifolia & L. \\
\hline Andromeda & Andromeda ligustrina & Muhl. \\
\hline Mountain Laurel & Kalmia latifolia & L. \\
\hline Sheep Laurel & Kalmia augustifolia & L. \\
\hline Pale Laurel & Kalmia glauca & Ait. \\
\hline Great Laurel & Rhododendron maximum & L. \\
\hline White Honeysuckle & Azalea viscosa & L. \\
\hline Purple Azalea & Azalea nudiflora & L. \\
\hline Rodora & Rhodora Canadensis & L. \\
\hline Black Alder & Ilex verticillata & Gray. \\
\hline Smooth Winterberry & Ilex lævigata & Gray. \\
\hline Spice Bush & Lindera Benzoin & Meisner. \\
\hline Moose Wood & Disca palustris & L. \\
\hline Sweet Fern & Comptonia asplenifolia & Ait. \\
\hline Wax-Myrtle & Myrica cerifera & L. \\
\hline Wax Work & Celastrus scandens & L. \\
\hline Fly Honeysuckle & Lonicera ciliata & Muhl. \\
\hline Mountain Fly Honeysuckle & Lonicera cærulea & L. \\
\hline Small Honeysuckle & Lonicera parviflora & Lam. \\
\hline Hazel-nut & Corylus Americana & Walt. \\
\hline Beaked Hazel-nut & Corylus rostrata & Ait. \\
\hline Mountain Holly & Nemopanthes Canadensis & D. C. \\
\hline Bearberry & Arctostaphylos Uva-ursi & L. \\
\hline Green Brier & Smilax rotundifolia & L. \\
\hline Green Brier & Smilax gluca & Walt. \\
\hline Shining Rose & Rosa lucida & Ehrh. \\
\hline Swamp Rose & Rosa Carolina & L. \\
\hline Purple Flowering Raspberry & Rubus odoratus & L. \\
\hline Black Raspberry & Rubus occidentalis & L. \\
\hline High Blackberry & Rubus Villosus & Ait. \\
\hline Running Blackberry & Rubus hispidus & L. \\
\hline Dwarf Blackberry & Rubus triflorum & Rich. \\
\hline Dewberry & Rubus Canadensis & L. \\
\hline Bush Honeysuckle & Diervilla trifida & L. \\
\hline Red Raspberry & Rubus strigosus & Michx. \\
\hline Shrubby Cinquefoil & Potentella fruticosa & Linn. \\
\hline
\end{tabular}


